\title{
Integration of Quantum Computing with IoT
}

\author{
Aman Kaushik, Rohit Narwal
}

\begin{abstract}
The Internet of Things (IoT) is one of the emerging fields which has been developing day by day since 1990 but still having issues like security, privacy, data theft and server requirements to deal with big data. Quantum computing solves most of the problems in IoT but it is still under research stage and can take about a decade or two to get publically available for public use. In this paper, we begin with general introduction on IoT and Quantum Computing and continue on with problems that can be faced during integration of IoT and Quantum Computing. After that, we will also point out the solution to encounter these integration issues in context of efficiency and cost. Finally, we point out the benefits of integration of both fields to existing IoT network architecture.
\end{abstract}

Index Terms: Decoherence, IoT, Entanglement, Qubits, Superconductors

\section{INTRODUCTION}

The Internet of Things (IoT) is among one of the most profound technologies that disappear completely from our observation, yet it weaves itself into the fabric of daily life. This is becoming a well-known concept across many organisations including a common man's everyday life. The development of the Internet of Things (IoT) is driven by the needs of large corporations to follow up their production chain which they are embedded in. The Internet of Things (IoT) was a major shift in the IT sector back then in the 90's when it first came into practical use. But now, this technology is the soul of most of the networks today whether it is a home network or a corporate network, Internet of Things (IoT) can be now seen in the medical sector, education sector, industries, and at any place where there is a need of a large network of embedded devices. Talking precisely, Internet of Things (IoT) is a system of interconnected computing devices, mechanical and digital machines or objects that are able to transfer data without requiring human-to-computer or human-to-human interaction. IoT is basically used for automation to ease or to reduce human interaction. In an IoT network, the devices or things (sensors and actuators) work together to solve a problem which requires more accuracy, regular computing and less human interaction. As there can be thousands of nodes participating in the network, they have to have some common requirements because there is a less chance that they are just deployed in the arena and someone regularly monitor or physically take care of them.

Revised Manuscript Received on April 17, 2020.

* Correspondence Author

Mr. Aman Kaushik*, Assistant Professor, Chandigarh University, Gharuan (India) aman.cse@cumail.in

Rohit Narwal, B.E. CSE (IoT), Chandigarh University, Gharuan (India) ait.17bcs4637@gmail.com

(C) The Authors. Published by Blue Eyes Intelligence Engineering and Sciences Publication (BEIESP). This is an open access article under the CC BY-NC-ND license (http://creativecommons.org/licenses/by-nc-nd/4.0/)
This results in issues like data security, monitoring, direct internet connection and much more. To mitigate these issues, the network can be assembled and integrated with the Quantum Computing technology.Quantum Computing is the use of quantum properties like entanglement and superposition to perform computation. The idea of exploiting quantum properties like entanglement and superposition to perform computation first came into place in 1960 when a research physicist named Stephen J. Wiesner invents Conjugate Coding and then later in 1973, when a Soviet and Russian mathematician named Alexander Holevo publish a paper showing that $n$ number of qubits can hold more information than $n$ number of classical bits. Due to less advancement in quantum systems at that time and some debates in physicists that it's not possible at all to design a system that can handle quantum particles in such a manner to do any kind of computation on them. But the research continues and it becomes practical in 2000 when the first working 5-qubit Nuclear Magnetic Resonance (NMR) computer was put through its paces at Technical University of Munich. About 15 years later, major tech giants like Google, IBM, Microsoft get in the competition of achieving Quantum Supremacy. In 2017, IBM launches its first prototype of Quantum Computer (IBM Q) for public access which can be accessed via the cloud. The research work is going on so fast that soon after maybe 10 years everyone has a small quantum device in their pockets just like smartphones. But for now, the work is still under progress and can take upto 10 or maybe 20 years to get into the common person's hand. Theoretically, if the architecture and the communication protocol (i.e entanglement) of quantum systems remain just as we saw today, it can be integrated in the IoT networks using the proposed architecture to make them secure and very fast as compared to what we have now today. Replacing every node from an IoT network and using a quantum device will not be practical as it can cause too much overhead and handling this much entanglement will be too messy and very complicated. If there are 10 quantum devices in an IoT network, every device must be able to communicate to each other, and to do so every device must hold qubits which are entangled to other devices. As only two qubits are entangled with each other at a time, one device has to have $2 n-1$ number of qubits where $n$ in this case is 10 , so each device must hold 19 qubits in order to communicate with each other. Qubits are not the same as classical bits on which today's technology works. Qubits are very sensitive to noise (change in temperature, pressure and any environmental phenomenon) and can easily lose their entanglement and superposition. Also the quantum system requires a very isolated and cold environment, it is not possible to isolate every device as they can be as small as a bottle cap and thousands in number distributed far away from each other.

Published By: 
To deal with this issue, the proposed architecture will use a quantum server as a gateway and can be easily implemented by just replacing the router with quantum server. The server will handle all the quantum work by converting the quantum input into classical bits as an output and forward the classical bits to desired IoT device.

\section{LITERATURE SURVEY}

1. Internet of Things (IoT): A Literature Review (May 2015)

IoT is a buzzword in Information Technology, that will be the future which will transform the real world objects into intelligent virtual objects. The IoT technology aims to unify everything in a common infrastructure, providing not only the control but also keeps us informed of the state of things. It is a branch that deals with the study of virtual things (or devices) connected in a network with the ability to take and sense any action around them.

2. The Quantum Limit to Moore's Law (July 2008)

Moore's Law gives us a predicting growth of computing power from increased density of transistors on integrated circuit (IC) chips. Many believe that trend will continue; however, quantum computing may define a future limit to electronic miniaturization and everincreasing computing power. Heisenberg uncertainty principle in terms of the Compton wavelength of particle physics suggests such a boundary, even for advances in quantum computing.

3. Quantum Computing with superconductors (Sept 2004)

Superconductive technology is one of the most promising approaches to computing in quantum systems. It offers devices with little dissipation and electrometers for state readout. Superconductors are the key ingredient in quantum computing as qubits can only be controlled with superconductors. The spin, position and orientation of qubit can be made possible only with the use of superconductors till now.

\section{STATEMENT OF PROBLEM}

In today's world many IoT systems are designed by considering various environmental parameters. Existing systems in any of the IoT networks today can easily be deployed at any place on earth and can fairly work without any hindrance in environmental as well as device parameters. But this will not be the case if we use quantum devices. A quantum device is dependent on qubit (quantum bit) for it's calculation and keeping the qubits in their original state is very difficult yet. Only a tiny bit of disturbance from neighbour qubit can totally hinder the original state. Qubits are mostly just ions or particles which are kept in superposition and entanglement simultaneously to perform operations. Qubits can easily lose their original state if disturbance like magnetic field, temperature, pressure etc. as well as if any other particle are introduced in the environment. Due to these factors, the quantum system requires special hardware to hold them. As for today, the hardware is known as a quantum data plane which has operations like holding the qubits in place and performing gate operations and control and measurement plane for controlling and measuring the qubits. These qubits are stored in an ion trap at milli-kelvins to avoid outer environment disturbance. Reaching this low temperature is itself a huge challenge even in research conditions, so it will be impractical to think using any kind of quantum system at home or for general use.

Fig. 1 shows the possible architecture that can be implemented via integrating every IoT node with quantum node. During implementation of today's quantum computing in IoT will introduce some of the problems which are stated below.

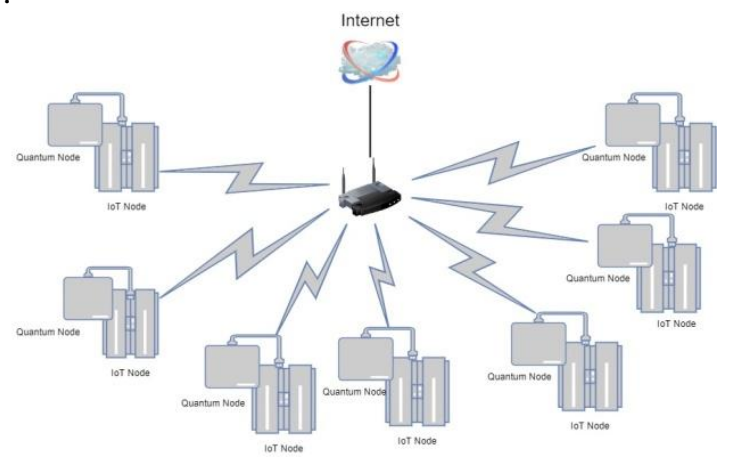

Fig. 1 Possible system

\section{Problem 1. Heavy Machinery}

If the present system is used, it will require a very large and heavy machinery to connect irrespective of the size of the network. This will be a problem if there's a small network which consists of just a handful of devices. As there has to be one quantum system present for just a single device which can be too impractical and over powered for today's small devices like CCTV, thermostat, alarms etc. which are very common in almost every IoT network. Introducing heavy machinery also means constant power supply and today's quantum computer uses 25 kilowatts per year to run. This much power can cause a tremendous amount of heat causing almost every IoT device to fail. Also, this is counter to the principle for IoT where devices run on even a single button cell of 3 volts. Additionally, it will not be cost efficient to afford that number of devices. Even after all of this if the system is designed, it will not be easy to deal with this much large and multiple systems.

\section{Problem 2. Inefficiency And Wastage Of Storage}

IoT networks are composed of small devices with a handful of Mhz of processing power which even wake up only during certain events, so most of the time devices are in sleep mode. Processing power is on peak just when it deals with huge data, which in IoT case is done mostly on remote devices. With the existing model, there will be too much wastage of resources as the quantum computing will be integrated on client side not on the server side where data operations takes place. Even in existing model, there is negligible need for processing, the only part where computation occurs is after all the data is gathered and processed. It will be beneficial to perform tasks which include machine learning or deep learning like part on server side, but quantum computing technology is not yet that advanced to do such tasks and even if it can be in some years, it still have to be done on server side or on controlling node not on the embedded device in the network. Apart from digital resources, the money will also be the issue for such a large and expensive system.

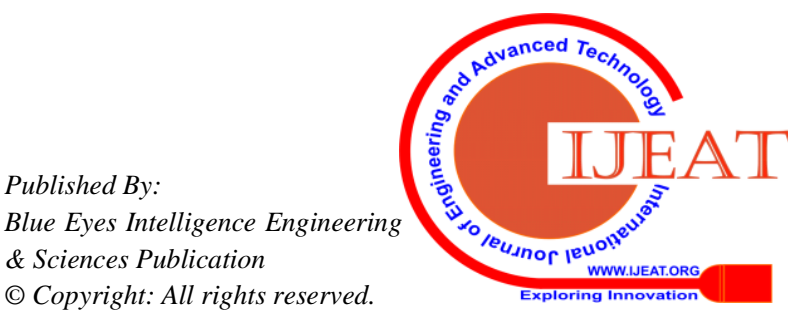




\section{Problem 3. Stability:}

Quantum systems are extremely unstable to surroundings since any interaction or measurement leads to collapse of the state function.

This phenomenon is called decoherence. The problem with handling a large number of qubits is that the system becomes too unstable and collapses. More the number of qubits the more it becomes vulnerable to decoherence. Another factor which results in an unstable system is that IoT devices remain in sleep mode for a very long time until a certain event happens, and qubits can't sustain in entangled states for such a long time. Due to this, the system has to generate entangled qubits again and again for every device in the IoT network every time the system suffers with decoherence which becomes very unstable in context with the reliability of the system.

\section{Problem 4. Hindrance In Functionality Of Iot Devices}

The main problem which has to be in light here is that; If the system is kept under isolation, how does it even do its job? Jobs like CCTV, environment sensing, forest monitoring and being aware of its surroundings is must for not all but most of the IoT systems. This is further a problem, if the system will be isolated how does the system even do for what it's being programmed for. Either the system has to work without isolation or if the isolation is removed, then the qubits will not sustain and the system has to work without the presence of any processing unit which both are not possible in this case.

\section{Problem 5. Qubit's Supply To The System}

Quantum systems are a completely different system than our existential classical systems; to send data, one seperate entangled qubit is required on the receiver side and for every new receiver the system has to maintain a unique qubit for each one new device because entanglement can take place between two entities only. As shown in Fig. 2, the quantum server has to handle four qubits for four of the devices connected to it.

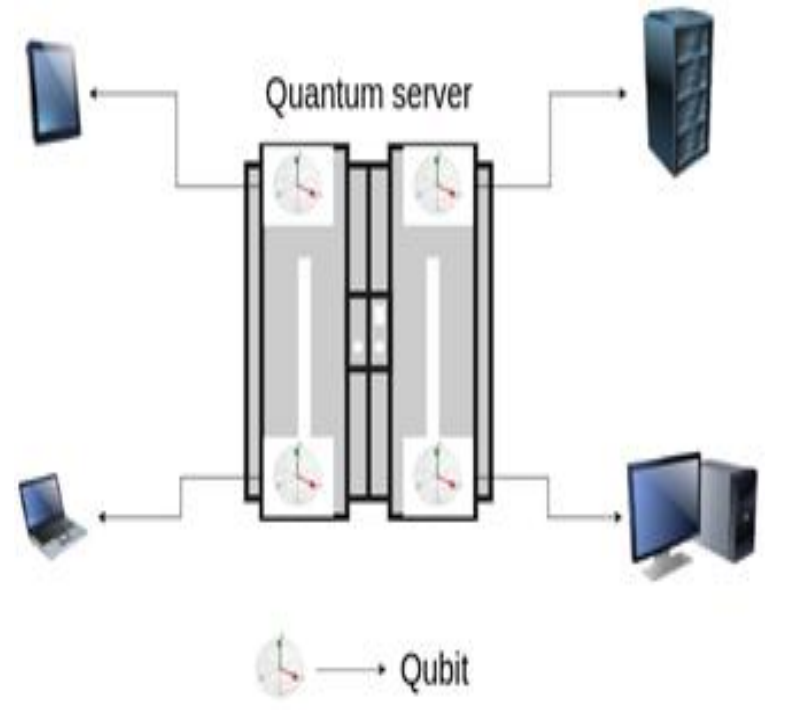

Fig.2 Qubits distribution in possible system

. Comparing this scenario with a real world IoT network where not only the server but the devices itself are connected to each other to make such a complex network. In that case, it gets even more complicated. If we assume a network of " $\mathrm{N}$ " number of interconnected devices, then each one of them has to have $2 \mathrm{~N}$ qubits to maintain the connection to each other. Having 2 qubits instead of 1 is not a problem, but in a network of even 20 devices, every device must have 40 qubits and the server have to create and distribute $40 * 20$ qubits for the network and as sustainability time for qubits is nearly 2 seconds, which makes the system unable to communicate

\section{PROPOSED SOLUTION}

For now the quantum computer is just for research purposes but as with every technology, one day it will be too common that it becomes part of everyday life just like IoT. But for now, they cost about $\$ 10$ billion in just hardware, far from the reach of common use for an average human. In future when it becomes small and is available to everyone, at that time it will be a new era of quantum technology. It will enforce mostly every field where computing requires including healthcare, military, communication and so on. IoT will also be the one which will get a new turn with quantum technology. As the limitations and hurdles are discussed above, there is still a way to integrate both IoT and Quantum technology and make the best out of them. Instead of making every device in an IoT network a quantum operated device, there can be a main server (quantum) just like a gateway which performs all the quantum operations and passes on the classical information to the IoT network. All the quantum operations will be on the server side; the generation and measurement of qubits, isolation of quantum system, communication to outer networks all of that will be managed by a core server as shown

in Fig. 3.

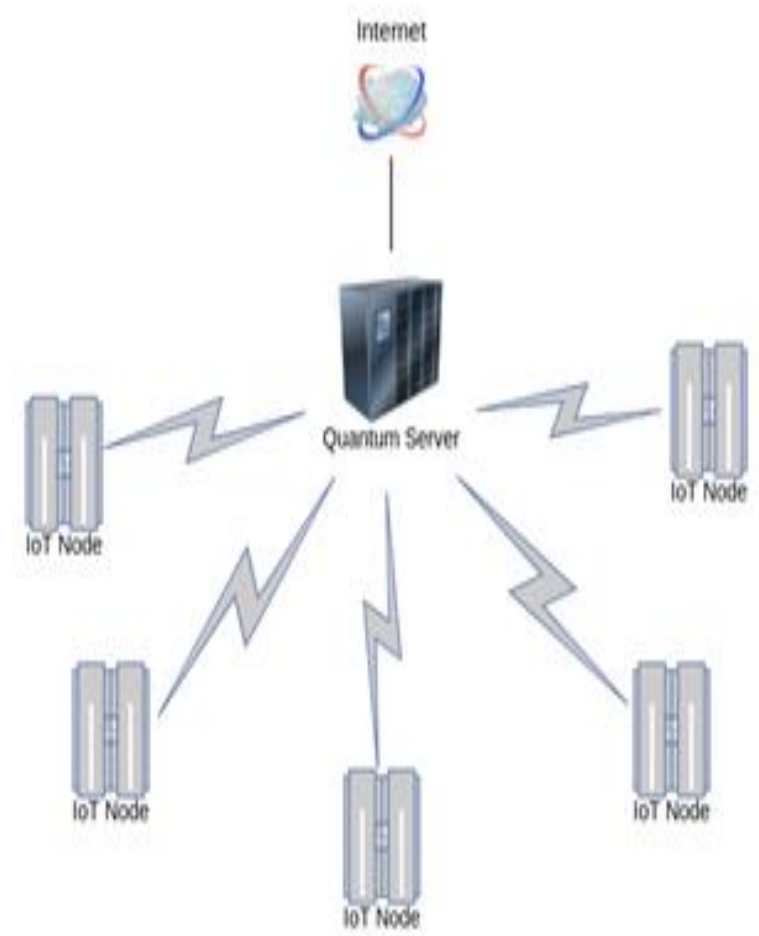

Fig. 3 Proposed systemx

The above figure shows the system that can be easy and efficient to implement as compared to the possible system as shown in Fig. 1. The flow of data in the proposed system will be as shown in Fig. 4.

Published By:

Blue Eyes Intelligence Engineering

\& Sciences Publication

(C) Copyright: All rights reserved.

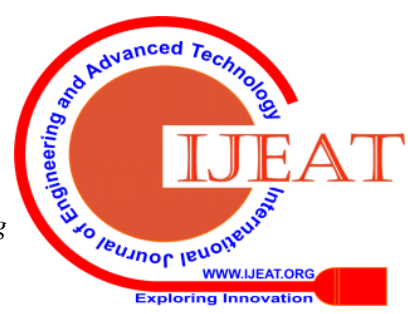




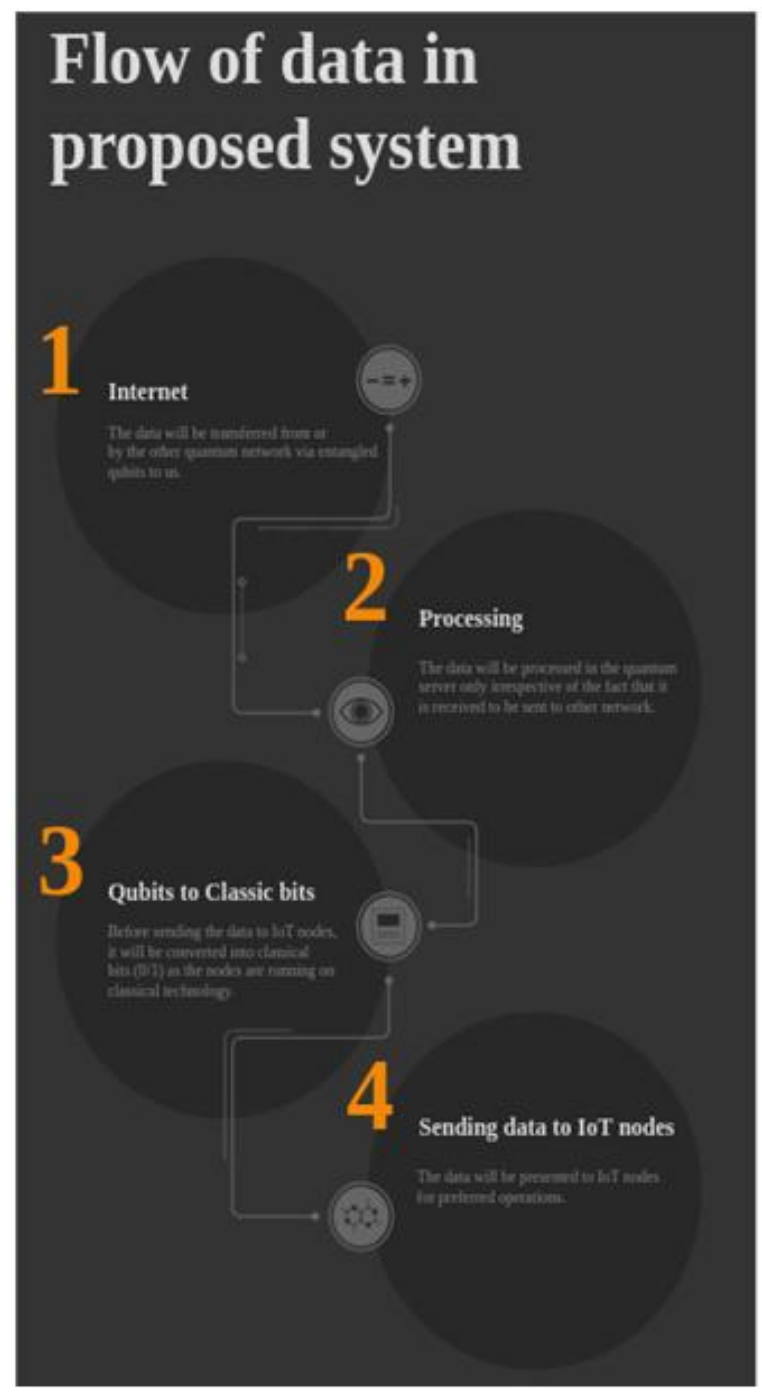

Fig. 4 Data flow in proposed system

In the proposed system, the quantum server will work as a gateway for the IoT network. All the data will be passed via the quantum server. Only the quantum server will have qubits and the rest of the IoT network will run on classical systems as it is running currently. The data pass to and fro via the quantum server only. The quantum server will process and transfer data via help of qubits in it. It will manage the generation and maintenance of qubits. When the data comes to the quantum server, it will process the quantum data and then convert it into classical bits. The classical bits are then sent to the IoT network which consists of devices which operate on classical bits ( 0 or 1 ). And when the data have to be sent to another network, the devices send classical data to the quantum server, the server will process the data and send it via the entangled qubits in it. The server can also be used to train and run machine learning models in itself only which will eliminate the need of cloud services and the data will be on user side making privacy issues a myth.

\section{BENEFITS}

The proposed system will have following benefits over the possible system:-

- No issue of backward compatibility or replacing the whole network and devices, just the gateway will be replaced with proposed quantum system.

- It will be a central point of failure and can easily be managed by the user.
- Data will remain on the user side eliminating the need of cloud and privacy issues.

- Can be stored in a single isolated environment without affecting the rest of the network.

- It will be far more secure than the present system as it is not possible to tamper quantum data by third parties as it results in collapse in entanglement state and the user can easily monitor the tamperming practices by the intruder.

- Machine learning, data analytics, AI (Artificial Intelligence) and much more can be done on the user side only as quantum computers can compute very complex problems in a very short time as compared to present classical computers.

\section{RESULTS}

To overcome the above discussed hurdles a new architecture for Quantum IoT network can be followed as shown in Fig.

5. It consists of four layers which have their own roles in the network as stated below:-

1. Application Layer:- This layer consists of application, monitoring system or end user system where the information finally meets the client. The client can send and receive commands to the next layer via qubits. (Qubits are transferred, not classical bits)

2. Quantum Teleportation Layer:- This layer only consists of quantum repeaters whose job is to maintain integrity of state of qubits being passed to it and forward qubits to next hop. Qubits can lose their entanglement state while traveling through large distances and to overcome this, we use quantum repeaters.

3. Quantum Network Layer:- This layer administers the quantum and classical data being transferred via local network and internet. It further consists of two parts:-

- Quantum Server:- It consists of quantum device which process and handle all the quantum data related operations and conversion of classical bits to qubits and vice versa.

- Gateway:- It will be the existing devices which act as a gateway like routers, which transfers classical bits from Physical layer to Quantum Server in Quantum Network Layer and vice versa.

4. Physical Layer:- All the IoT devices in a network will be the part of this layer. This layer remains the same as that of today's existing IoT network, with no difference. The devices will send classical bits to the gateway as usual but after that, the gateway sends those bits to the quantum server and they further get converted into qubits by the quantum server in Quantum Network Layer.

Published By:

Blue Eyes Intelligence Engineering \& Sciences Publication (C) Copyright: All rights reserved.

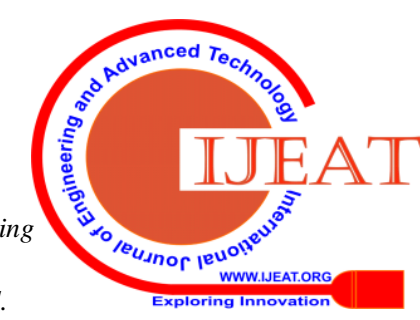




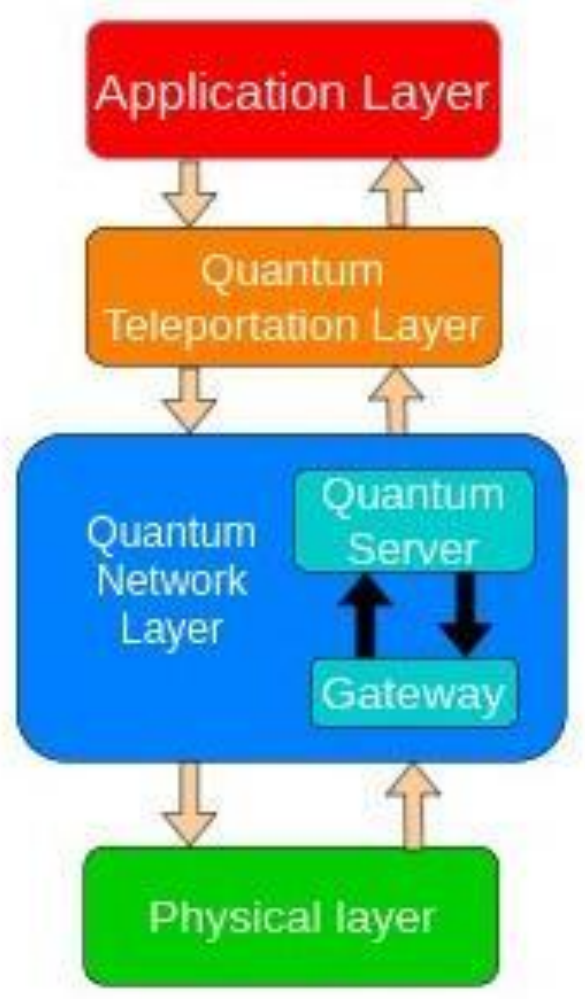

Fig. 5 Quantum IoT network architecture

\section{CONCLUSION}

IoT brings a technological change in our lives and becomes a part of everyday life. As the new technologies will emerge in our society, they open a pathway to improve the present one. Here quantum computing is the new opportunity for improving the existing IoT technology. With the help of the proposed system, quantum computing will be easily and efficiently implemented in IoT without the need of replacing the whole network making the existing system more reliable, efficient, secure and fast in terms of processing.

\section{REFERENCES}

1. D. Martell, "Intel's Moore muses on end of technology maxim" Reuters, Sep. 19, 2007

2. Detecting the spin of a single electron, Los Alamos National Laboratory, Aug. 12, 2004, news release.

3. Feng Xia1, Laurence T. Yang2, Lizhe Wang3 and Alexey Vinel4, "Internet of Things", DOI 10.1002/dac.2417, 2012; Available at International Journal of Communication Systems site: https://www.academia.edu/download/40650301/danainfo.acppwiszgm k2n0u279qu76contentserver.pdf

4. Zhi-Kai Zhang, Michael Cheng Yi Cho, Chai-Wei Wang et al. "IoT Security: Ongoing Challenges and Research Opportunities" in 17-19 Nov. 2014, IEEE 7th International Conference on Service-Oriented Computing and Applications, pp. 2163-2871.

5. IBM's quantum cloud computer goes commercial, International weekly journal of Science Nature, 06 March 2017, news release.

6. Graham P. Collins, "Computing with Quantum Knots", JSTOR, Vol. 294, No. 4, pp 56-63, April 2006

7. Rodney Van Meter, Thaddeus D. Ladd, Austin G. Fowler and Yoshihisa Yamamoto, "Distributed Quantum Computation Architecture using semiconductor nanophotonics", International Journal of Quantum Information, Vol 08, No. 01n02, pp. 295-323, 2010; Available on World Scientific site: https://www.worldscientific.com/doi/abs/10.1142/S021974991000643 5

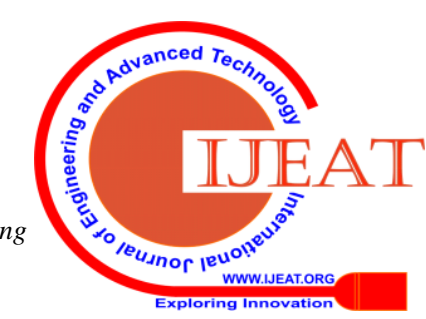

